FERMILAB-CONF-04-324-AD

Resistive-Wall Instability at Fermilab Recycler Ring K.Y. Ng 


\title{
Resistive-Wall Instability at Fermilab Recycler Ring
}

\author{
K.Y. Ng \\ Fermi National Accelerator Laboratory, P.O. Box 500, Batavia, IL 60510
}

\begin{abstract}
Sporadic transverse instabilities have been observed at the Fermilab Recycler Ring leading to increase in transverse emittances and beam loss. The driving source of these instabilities has been attributed to the resistive-wall impedance with space-charge playing an important role in suppressing Landau damping. Growth rates of the instabilities have been computed. Remaining problems are discussed. [1]
\end{abstract}

Keywords: resistive wall, transverse instability PACS: $41.75 . \mathrm{Lx}$

\section{TRANSVERSE INSTABILITIES}

The Fermilab Recycler Ring stores and cools antiprotons at $E=8.938 \mathrm{GeV}$ for injection into the Tevatron. Since the beginning of 2004, transverse instabilities have been reported in antiproton beams with the signature of a sudden increase in transverse emittances and a small loss in beam intensity. The first such documented transverse instability was observed on February 19, 2004, [2] when a stochastically cooled antiproton beam of intensity $126 \times 10^{10}$ and length $7.3 \mu$ s stored between two barrier waves took a sudden jump of horizontal and vertical 95\% normalized emittances from $\sim 7 \pi$ to $\sim 15 \pi \mathrm{mm}-\mathrm{mr}$ accompanied by a beam loss of $\sim 1 \times 10^{10}$.

The first culprit to blame was ion-trapping and the stray fields of the Main Injector that shares the same tunnel. However, the possibility of ion-trapping was dismissed after two experiments. [3] The first on June 9 was a transverse instability of similar nature induced on an antiproton beam of intensity $N_{b}=28 \times 10^{10}$, full length $t_{b}=3.5 \mu \mathrm{s}$, rms energy spread $\sigma_{E}=3 \mathrm{MeV}$, and $95 \%$ horizontal and vertical normalized emittances $3 \pi \mathrm{mm}-\mathrm{mr}$ by lowering the vertical chromaticity from $\xi_{y}=-2$ to zero. The difference signals of the beam were sampled with a vertical beam-position monitor at the rate of $125 \mathrm{MHz}$. The FFT shown in Fig. 1 reveals excitation of the betatron sidebands rolling off very slowly until roughly at the 70th harmonic. If the instability were driven by trapped $\mathrm{CO}^{+}$ions, the ion-in-beam bounce frequency which peaked at $\sim 100 \mathrm{kHz}$ would cluster only around the first and second harmonics (90 and $180 \mathrm{kHz}$ ). The second experiment on July 9 was the observation of a similar transverse instability induced on a stored proton beam which could not trap positive ions. The proton beam was of intensity $N_{b}=43.9 \times 10^{10}$ filling the whole ring circumference of $11.13 \mu \mathrm{s}$, with $95 \%$ horizontal and vertical normalized emittances $6 \pi \mathrm{mm}-\mathrm{mr}$ before the instability blew them up to more than double together with $16 \%$ loss of beam intensity. The difference signal indicated that the growth time was about 500 to 1000 turns or 5.6 to $11 \mathrm{~ms}$. The rms energy spread was

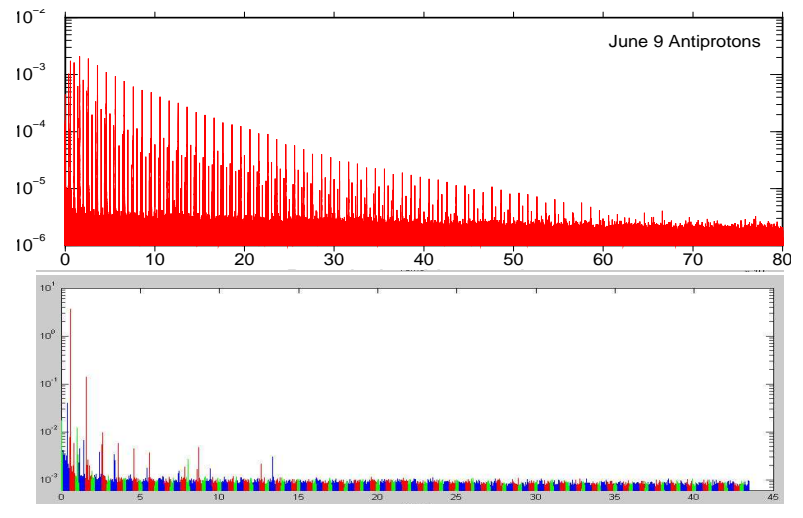

FIGURE 1. Top: Excitation of betatron sidebands of the first 80 revolution harmonics monitored at the split-tube BPM VP522 during the antiproton beam instability induced on June 9. Bottom: Excitation of betatron sidebands of the first 45 revolution harmonics of the proton beam on July 9. The preamp at VP522 has flat response from $10 \mathrm{kHz}$ to $10 \mathrm{MHz}$.

$1 \mathrm{MeV}$ and did not change throughout the instability. The FFT in Fig. 1 shows the lower betatron sidebands excited very much more than the upper sidebands.

\section{DISPERSION RELATION AND IMPEDANCES}

For Gaussian-distributed energy spread, the dispersion relation governing Landau damping can be expressed as

$$
U+i V=-\frac{e I_{0} \beta^{2} c Z_{1}^{\perp}}{4 \sqrt{2 \pi} v_{y} \omega_{0} \sigma_{E}\left|S_{y}\right|}=\frac{1}{w\left(z_{1}\right)},
$$

where $S_{y}=\xi_{y}-\eta\left(n-\left[v_{y}\right]\right)$ for $n=1,2, \cdots$ is the effective chromaticity, $\eta=-0.008812$ is the slip factor, the nominal betatron tunes are $v_{x, y}=25.425 / 24.415$ with $\left[v_{x, y}\right]$ their decimal parts, and $w\left(z_{1}\right)$ is the complex error function. The collective eigenfrequency is

$$
\Omega=n \omega_{0}+\omega_{y}+\frac{\sqrt{2} \sigma_{E}\left|S_{y}\right| \omega_{0}}{\beta^{2} E} z_{1},
$$

and the imaginary part gives the growth rate. Here, $\omega_{y} / \omega_{0}$ is the bare vertical betatron tune plus the inco- 
herent tune shift, $n$ is any revolution harmonic, positive or negative, and $\beta$ and $\gamma$ are Lorentz parameters.

Besides space-charge, the transverse impedance receives most contribution from the resistive walls of the vacuum chamber, which is made of stainless steel with an elliptical cross section of diameters $3.75^{\prime \prime} \times 1.75^{\prime \prime}$. The transverse resistive-wall impedances are

$$
\begin{aligned}
& Z_{1}^{H}=(1-i) 11.79\left|n-\left[v_{x}\right]\right|^{-1 / 2} \mathrm{M} \Omega / \mathrm{m}, \\
& Z_{1}^{V}=(1-i) 21.92\left|n-\left[v_{y}\right]\right|^{-1 / 2} \mathrm{M} \Omega / \mathrm{m},
\end{aligned}
$$

with $n=1,2,3, \cdots$ denoting the excitation of the lower betatron sidebands $n-\left[v_{y}\right]$, which are also commonly known as the $(n-Q)$ lines.

The proton beam had been scraped heavily leaving behind about only one-half of its initial intensity. We therefore approximate the transverse distribution as uniform with a vertical radius $a_{y}=\sqrt{\beta_{y} \varepsilon_{N 95 \%} /(\gamma \beta)}$ and a similar horizontal radius $a_{x}$ including dispersion. The transverse space-charge impedance is given by

$$
\left.Z_{1}^{V}\right|_{\text {spch }}=i \frac{2 Z_{0} v_{y}}{\gamma^{2} \beta^{2}}\left\langle\frac{\beta_{y}}{a_{y}\left(a_{y}+a_{x}\right)}\right\rangle
$$

where $Z_{0} \approx 376.7 \Omega$ is the free-space impedance and $\beta_{y}$ is the vertical betatron function. At initial $95 \%$ normalized emittance $\varepsilon_{N 95}=6 \pi \mathrm{mm}$ - $\mathrm{mr}$ in both transverse planes and integrating over the Recycler Ring using the most recent lattice, we get $\left.Z_{1}^{V}\right|_{\text {spch }}=161.8 \mathrm{M} \Omega / \mathrm{m}$.

For the antiproton beam, the transverse distribution is bi-Gaussian because of stochastic cooling. The equivalent-uniform-distribution beam radii of a bi-Gaussian distribution are $a_{x, y}=\sqrt{2} \sigma_{x, y}$ with vertical rms radius given by $\sigma_{y}=\sqrt{\varepsilon_{N 95} \beta_{y} /(6 \beta \gamma)}$ and a similar horizontal radius including dispersion. At $\varepsilon_{N 95}=3 \pi \mathrm{mm}-\mathrm{mr}$, the space-charge impedance is $\left.Z_{1}^{V}\right|_{\text {spch }}=959 \mathrm{M} \Omega / \mathrm{m}$, which is very much larger than the resistive-wall impedance in magnitude.

\section{INDUCED PROTON INSTABILITY}

For the experiment on the proton beam instability, the equi-growth contours are drawn in the $U-V$ plane in Fig. 2. The $U-V$ 's (or $-Z_{1}^{V}$ ) of the lower betatron sideband excitations $(n-Q)$ are shown as circles for various vertical chromaticities $\xi_{y}$. We see that $\xi_{y}=-0.773$ is required to stabilize the beam. Notice that Landau damping is significant only in the curved part of the contours. The growth times of $(1-Q)$ and $(2-Q)$ are found to be 51 and $84 \mathrm{~ms}$, respectively at $\xi_{y}=0$ with the aid of Eq. (2), compared with 75 and $105 \mathrm{~ms}$ in observation. The growth rates of all the unstable modes are calculated and are shown in Fig. 3. We find 59 lower-frequency modes unstable when $\xi_{y}=0$. However, in the FFT plot of Fig. 1, less than 20 unstable modes are observed.

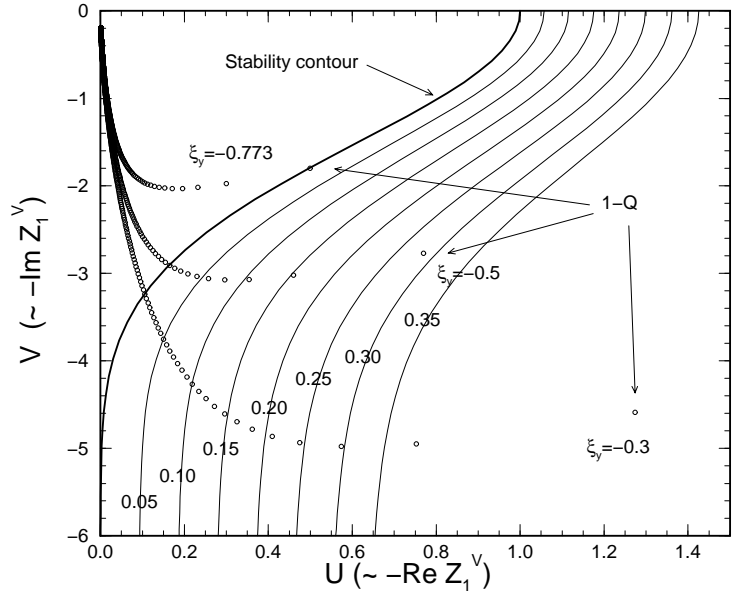

FIGURE 2. $U-V$ values of lower sidebands of proton beam at various chromaticities along with the equi-growth contours.

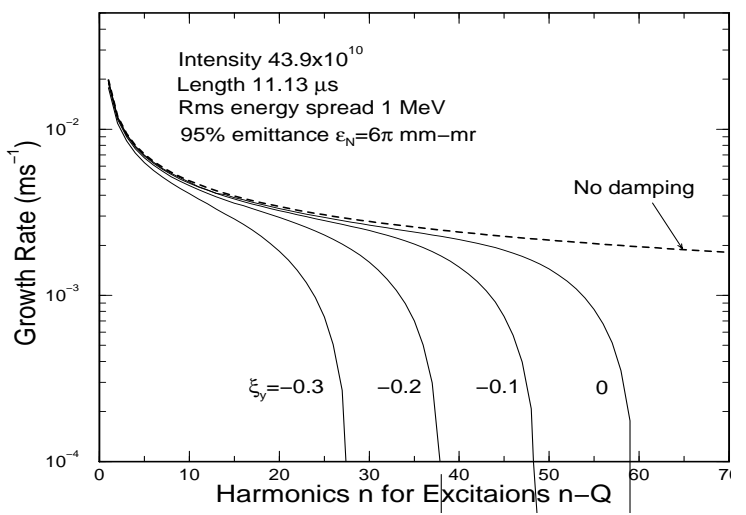

FIGURE 3. Theoretical growth rates of $(n-Q)$ excitations at various chromaticities.

\section{INDUCED ANTIPROTON INSTABILITY}

The instability of the antiproton beam was analyzed in the same way. The equi-growth contours are shown in Fig. 4 at $\xi_{y}=0,-0.5,-0.1$, and -0.2 . The growth rates of the excitation of the lower sidebands are computed from the dispersion relation and are depicted in Fig. 4. Theory predicts the first 12 sidebands unstable at $\xi_{y}=-2$. However, antiproton beams are always stable in operation at $\xi_{y}=-2$. Theory also predicts the first 216 modes unstable at $\xi_{y}=0$, while experimental observation gives about only 70 according to Fig. 1 .

\section{DISCUSSIONS}

(1) Solution of the dispersion relation agrees qualitatively that the transverse instabilities observed at the Recycler Ring were driven by resistive-wall impedance. However, quantitatively, the computed growth rates appear larger than observed, because not so many sidebands are recorded unstable in Fig. 1. Although nonlin- 


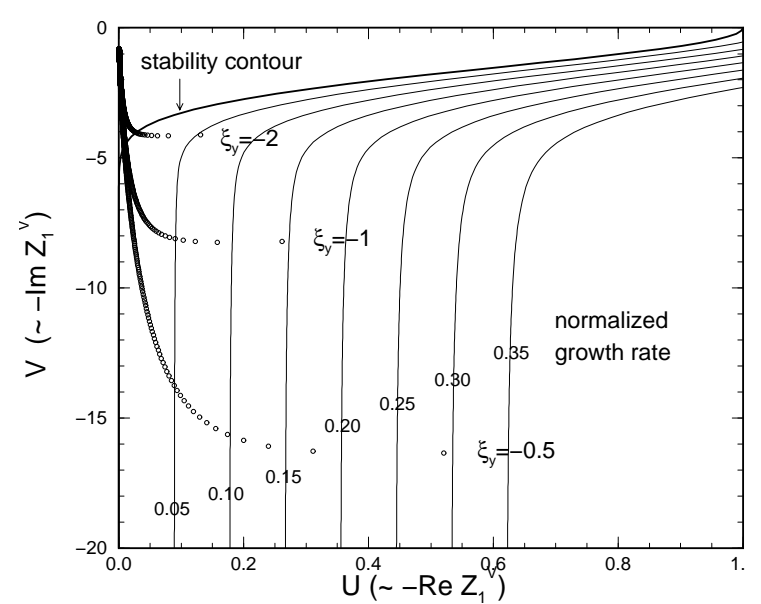

FIGURE 4. $U-V$ values of lower sidebands of antiproton beam at various chromaticities along with the equi-growth contours.

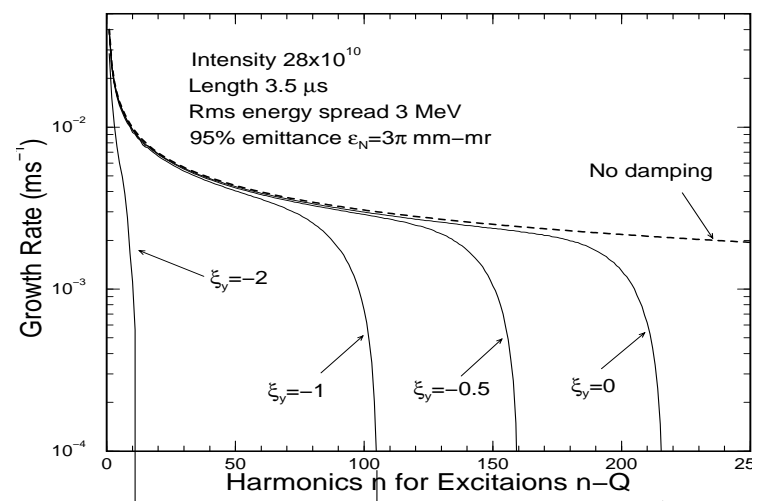

FIGURE 5. Computed growth rates of the excitation $(n-Q)$ at chromaticities $\xi_{y}=-2,-1,-0.5$, and 0 .

ear elements contribute additional tune spreads, unfortunately, the Recycler lattice shows that, like the incoherent space-charge tune spread, they are in the negative direction away from the coherent excitation and do not help stabilize the beam. The higher unstable modes, however, have very slow growth rates, and it is possible that their excitations were submerged in the noise background, implying that random noise might provide damping.

(2) We see in Fig. 4 that the $U$ value (or $R e Z_{1}^{V}$ ) of the $(1-Q)$ mode already resides within the stability criterion at $\xi_{y}=-0.5$. It is the space-charge that shifts the mode outside the stability contour by giving it a large $V$ value. The incoherent space-charge tune shift is related to the space-charge impedance by

$$
\left.\Delta v_{y}\right|_{\text {incoh }}=i \frac{e I_{0} R}{4 \pi \beta E v_{y}} Z_{1}^{V},
$$

which amounts to $-1.26 \times 10^{-4}$ for the proton beam when the instability occurs, where $R$ is the ring radius. Since $\xi_{y}=-0.773$ is required to stabilize mode
$(1-Q)$, it corresponds to a rms tune spread of $\sigma_{\Delta v_{y}}=$ $\left|S_{y}\right| \sigma_{E} /\left(\beta^{2} E\right)=0.87 \times 10^{-4}$. As shown in Fig. $6,2 \sigma_{\Delta v_{y}}$ will provide large enough spread to cover the coherent excitation making Landau damping functional.

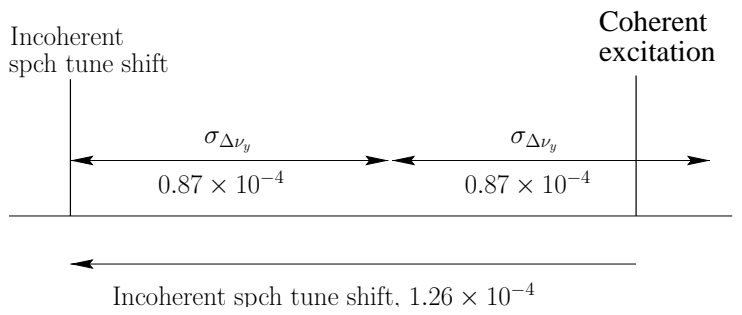

FIGURE 6. Drawing showing tune spread from chromaticity that overcomes the incoherent space-charge tune shift for the proton beam to arrive at Landau damping.

(3) We do not believe that the space-charge impedances have been overestimated. For the proton beam, although it had been heavily scraped, the transverse distribution would not have been uniform. Assuming a uniform distribution actually has led to a smaller space-charge impedance. For the antiproton beam, stochastic cooling made the distribution very bi-Gaussian, which had been verified by measurement using scrapers. [4] However, the formula for the space-charge impedance for a biGaussian distribution corresponds to the maximum incoherent space-charge tune shift, or for the very few particles at the center of the beam. Whether a smaller tune shift that corresponds to the average over the whole beam should be used requires more investigation.

(4) Skew quad SQ408 was discovered nonfunctional in late June. After its repair, no more sporadic transverse instabilities have ever been recorded. In the experiment on proton beam on July 9, SQ408 had to be turned off in order that the instability could be induced. It is possible that the beam gets jittered horizontally and needs coupling to the vertical to start instability. It is also possible that strong horizontal-vertical coupling moves the tune footprint to cover parametric resonances and starts the instability. At this moment the contribution of SQ408 to the instabilities has not been resolved.

\section{REFERENCES}

1. For more detailed discussion, see K.Y. Ng, "Transverse Instability at the Recycler Ring," Fermilab Report FN-0760, 2004.

2. All the experimental data reported were gathered by members of the Fermilab Recycler Ring Department.

3. Jim Crisp and Martin Hu, "Recycler Ring Instabilities Measured on 6/9/04," 2004, (unpublished); Martin Hu and Jim Crisp, "Recycler Instability Observed with Protons," 2004, (unpublished).

4. K. Gounder, M. Hu, and D. Broemmelsek, Transverse Beam Profile with Scraper Scans, Fermilab Internal Report, Main Injector Note 290, 2002. 